Gynäkologe 2018·51:452-453

https://doi.org/10.1007/s00129-018-4254-0

(c) Springer Medizin Verlag GmbH, ein Teil von Springer Nature 2018

CrossMark

Klaus Vetter ${ }^{1} \cdot$ Roland Zimmermann ${ }^{2}$

'Berlin, Deutschland

${ }^{2}$ Klinik für Geburtshilfe, Universitätsspital Zürich, Zürich, Schweiz

\title{
e-Gyn - Elektronik in Frauenheilkunde und Geburtshilfe
}

Sträuben sich da nicht die Nackenhaare, wenn uns ein Ausdruck wie e-Gyn begegnet? Was soll das nun wieder bedeuten? Worum es uns geht ist darzulegen, was neben Alltäglichem aus der Entwicklung der letzten Jahre hin zum aktuellen politischen Schlachtruf nach Digitalisierung in unserem Fachgebiet bedenkenswert ist.

Fünfzig Jahre nach dem mystifizierten 1968 und konsekutiven gesellschaftlichen Veränderungen, von denen die meisten in den Köpfen und nicht als Strukturveränderungen erfolgt sind, müssen wir uns einem anderen Wandel stellen: demjenigen der Ausbildung kommunikativer Netze, die keine Grenzen kennen und die unsere ärztliche Kommunikation, die der individuellen Beziehungen von Ärzten und Patienten, aber auch diejenige miteinander, absehbar - wenn nicht schon geschehen grundlegend verändert.

\section{\) Wir müssen uns dem absehbaren Wandel durch die fundamentalen Veränderungen der Kommunikation stellen}

Andréa Belliger führt uns umfassend in diese Entwicklung ein mit ihrem Beitrag: „Vom E im E-Learning und vernetzten Patienten. Digitale Transformation im Bildungs- und Gesundheitswesen.“

Auch wenn es ein Irrtum war, vom Tod der Printmedien auszugehen, zeichnen sich diese im Gegensatz zu elektronischen Medien durch Trägheit aus, die bei Büchern mehrere Jahre Verzögerung bedeuten. Das ist aktuell nur noch in be- stimmten Bereichen vertretbar, die von der allgemeinen Entwicklungsdynamik ausgenommen sind.

Näher an dem, was uns bekannt ist, sind die seit einigen Jahren in unserem Gebiet entstandenen elektronisch vermittelten und optimierten Möglichkeiten der Erweiterung von Aus-, Weiter- und Fortbildung durch Portale, wie GyneLearning. Roland Zimmermann und Tilo Burkhardt beschreiben in dem Artikel „GyneLearning - Eine Podcast-Fortbildungsplattform für Frauenärzte, Neonatologen und Hebammen als Ergänzung $\mathrm{zu}$ Live-Fortbildungsveranstaltungen“, wie aktuelles Fortbildungswissen auch denjenigen zugänglich gemacht wird, die an einer Veranstaltung nicht persönlich teilnehmen konnten und trotzdem à jour sein möchten.

Einen anderen Weg der elektronisch vermittelten interaktiven ärztlichen Online-Fortbildungsplattform ist die bewährte Plattform GynToGo. Thilo Gröning und Babett Ramsauer beschreiben in ihrem Artikel „Online-Fortbildung von GynToGo zu UniversityToGo“, wie diese Plattform dazu geeignet ist, mehr Zuschauer zu aktivieren als es eine nationale Großveranstaltung vermag und dabei live und interaktiv ist.

Nicht zuletzt war eine der Hoffnungen bei der Einführung elektronischer Medien in die Medizin, als Ärzte davon zu profitieren und Freiheiten für die Patientenversorgung zu gewinnen, beispielsweise, indem einheitliche vernetzte Dokumentationssysteme u. a. eine ärztliche „Doppeldokumentation“ unnötig werden lassen könnten. Leider ist das ganze Gegenteil zu beobachten: In Klinik und Pra- 
xis wird zunehmend mehr Zeit patientenfern für Dokumentation verbraucht. Schuld daran sind nicht nur die fehlende Ost-West-Einigung auf Laborstandards, wie das SI-System (SI: Système International d'Unités), sondern Uneinigkeiten an fast allen Schnittstellen.

Der letzte Beitrag ist dem Thema „Daten in Klinik und Praxis - was ist wichtig?" gewidmet, und Sylvia Thun zeigt auf, wo es trotz e-Health-Gesetz und geplanter elektronischer Patientenakte nach wie vor erheblich harzt, was getan werden sollte und worauf aktuell bei der Anschaffung von IT-Systemen (IT: Informationstechnologie) zu achten ist. Ihren Gedanken wäre zu folgen, damit der Traum von der Verbesserung durch IT realisiert werden kann.

Selbstverständlich reicht das Thema e-Gyn weit über die hier vorgetragenen Aspekte hinaus, und es wird Aufgabe auch dieses gedruckten Fortbildungsmediums Der Gynäkologe sein und bleiben, dies zukünftig $\mathrm{zu}$ thematisieren. Wir freuen uns auf Anregungen aus der Leserschaft.

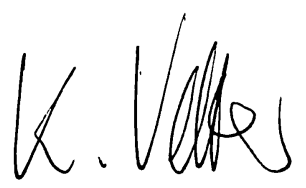

\section{Klaus Vetter}

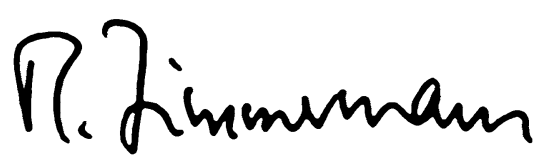

\section{Roland Zimmermann}

\section{Korrespondenzadresse}

Prof. Dr. med. K. Vetter,
MBA
Friedrichshaller Straße 7C,
14199 Berlin, Deutschland
kvetter@ngi.de

Interessenkonflikt. K. Vetter und R. Zimmermann geben an, dass kein Interessenkonflikt besteht.

\section{Kranke, P. (Hrsg.) Die geburtshilfliche Anästhesie}

Berlin Heidelberg: Springer-Verlag

2018, 1. Auflage, 682 S., 220 Abb.,

(ISBN: 978-3-662-54374-0), Hardcover 99,99 EUR, inkl. eBook

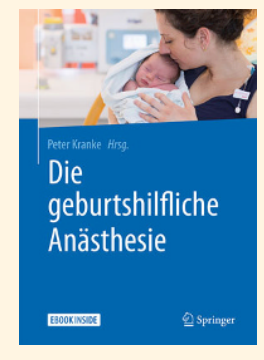

Peter Kranke und sein Team aus erfahrenen Autoren haben mit "Die geburtshilfliche Anästhesie" ein Fachbuch für den deutschsprachigen Raum geboren, welches

sich der Zusammenarbeit von Geburtshilfe und Anästhesie widmet.

Die mitwirkenden Anästhesisten, Geburtshelfer und Pädiater vermitteln verständlich und praxisnah die Grundlagen und Besonderheiten aus ihrem jeweiligen Blickwinkel und bieten sowohl dem Berufsanfänger als auch dem erfahrenen Kollegen die Möglichkeit, sich mit dem sensiblen Aufgabenbereich der Geburtshilfe intensiver zu beschäftigen. Schon beim ersten Durchblättern des Buches fällt die überzeugende Systematik und die ansprechende didaktische Gliederung ins Auge. Beginnend mit den Anfängen der Geburtshilfe werden die Meilensteine der modernen Geburtsmedizin sowie alle relevanten Allgemein- und Regionalanästhesieverfahren für geburtshilfliche als auch für nicht-geburtshilfliche Eingriffe während der Schwangerschaft dargestellt. Das physiologische und anatomische Grundlagenwissen ermöglicht ein Verständnis der komplexen Zusammenhänge und bietet das notwendige Rüstzeug für eine gute Versorgung von Mutter und Kind. Hierbei sind Themen wie die Pharmakotherapie in Schwangerschaft und Stillzeit genauso alltagsrelevant und gut verständlich erläutert, wie die Anpassung des Neugeborenen an das Leben außerhalb des Uterus. Kapitel zu Themenkomplexen wie Gerinnung und perinatale Blutung, Atemwegsmanagement und Intensivtherapie bei Schwangeren, sowie geburtshilfliche Komplikationen machen das Buch noch praxistauglicher.

Besonders gelungen sind die typischen Fallkonstellationen und die geburtshilflichen CIRS-Fälle, in denen das Lehrbuchwissen in den Alltag überführt und der professionel- le Umgang mit Fehlern lösungsorientiert dargestellt wird.

Auch fehlt es nicht an Handlungsempfehlungen und praktischen Tipps der erfahrenen Autoren, die verdeutlichen wie wichtig die sogenannten "soft skills" in diesem Bereich sind. Abgerundet wird das Buch durch eine Zusammenstellung von organisatorischen und medikolegalen Aspekten sowie nützlichen Internetseiten und Apps, die den Inhalt und Mehrwert der Tätigkeit in der geburtshilflichen Anästhesie erläutern.

Fazit: Ein wirklich praxisrelevantes und umfassendes Buch, welches in keinem Kreißsaal fehlen sollte.

T. Fink (Homburg) 